\title{
A simulator based on LED technology to study daylight on architectural scale models
}

\author{
Thierry Blandet $*^{*^{a}}$, Levin Erbilgin ${ }^{\mathrm{b}}$, Nicolas Hoerter ${ }^{\mathrm{c}}$, Nicolas Vergnes ${ }^{\mathrm{c}}$

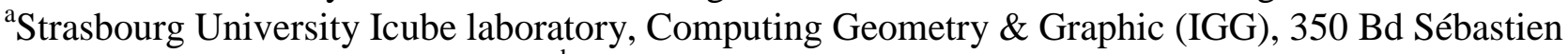 \\ Brandt, Illkirch, 67400, France; ${ }^{b}$ Strasbourg University Dept. of Architecture, 6-8 Bd Wilson \\ Strasbourg, France; ${ }^{c}$ Intern students, Master of engineering, University of Strasbourg
}

\begin{abstract}
The Strasbourg School of Architecture (ENSAS) developed a daylight simulator applied to architectural scale models. It reproduces both direct sunlight and diffusing light from the sky. Because LED technology offers a possible control of each source, it is no longer necessary to build the sky with a dome shape. Therefore we chose a cubic shape. RGB LED sources are fixed on a white panel behind a lambertian diffuser.

To reach a uniform lighting on the technical platform, the system has to be calibrated. The objective is to define the correction table to be applied to the artificial sky. After calibration in such a sky, the luminous flux of each source will have the same contribution to the centre of the experimental platform. Initial results have shown that the application of a theoretical correction function does not provide uniform illuminance. These first counter-intuitive results led to investigate several configurations which showed that the inter-reflection between the walls represent about $20 \%$ of the illuminance measured inside the simulator. Finally, because of these inter-reflections, the intensity of the LEDs in the corners should be reduced by around $10 \%$. To respect a lighting ratio of 6 between the sun and the sky, the intensity of the artificial sun has been adapted.
\end{abstract}

Keywords: LED, daylighting simulation, pixel mapping, sky models, artificial sky, daylight simulator, calibration

\section{INTRODUCTION}

The Strasbourg School of Architecture (ENSAS) developed its own LED daylight simulator applied to architectural scale models in order to use it for student training purposes. The main innovative choice was to achieve this simulator by using only LED technologies while it guarantees low power consumption with an optimal and tunable polychromatic light emission. This LED lighting system set our simulator at the cutting-edge of the technology from all other equivalent systems in the world since they only use halogen or filament lights which are not tunable white sources.

\subsection{General presentation}

Our daylight simulator is a system that allows the study of direct solar radiation completed with the luminous contribution of a configurable artificial sky. In this system, the model is placed on a circular technical platform. The sky has a cubic shape with the following inside dimensions: $4780 \mathrm{~mm}$ x $4780 \mathrm{~mm}$ x $3780 \mathrm{~mm}$ (width x length x height). 


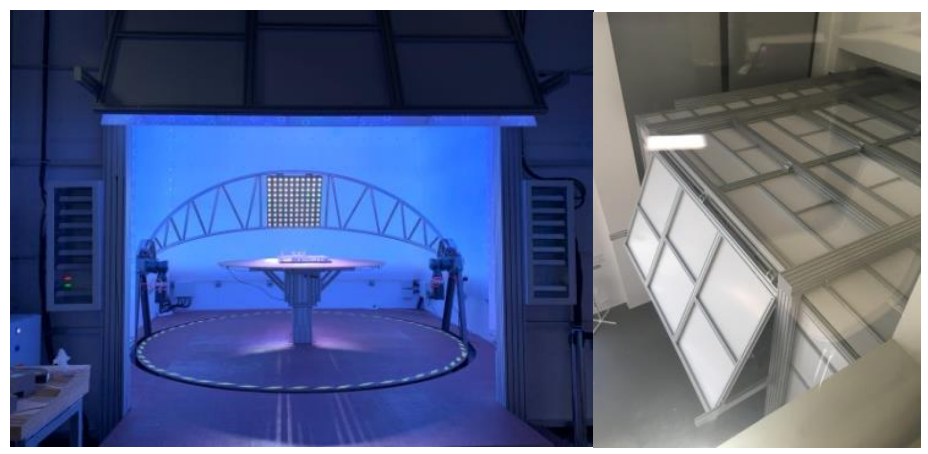

Figure 1: Picture of a model illumined by the artificial sun and the artificial sky in the cubic shape of our sun-sky simulator and top view of the simulator with its awning

The user defines the geographical position of the desired solar path as well as the day. During a typical 3 minutes' simulation, the sun goes from sunrise to sunset, follows its trajectory in the sky [1], the colour and intensity of the artificial sun and sky evolve in the simulated day as in reality. The sun and sky simulator has to be considered mainly as a qualitative device.

\subsection{State of the art of daylight simulators}

Daylight simulators applied to architecture models are developed since the middle of the twentieth century. Each laboratory develops its own simulators according to the current technologies and depending on the requirements expected on the results $[2,3,4]$. 


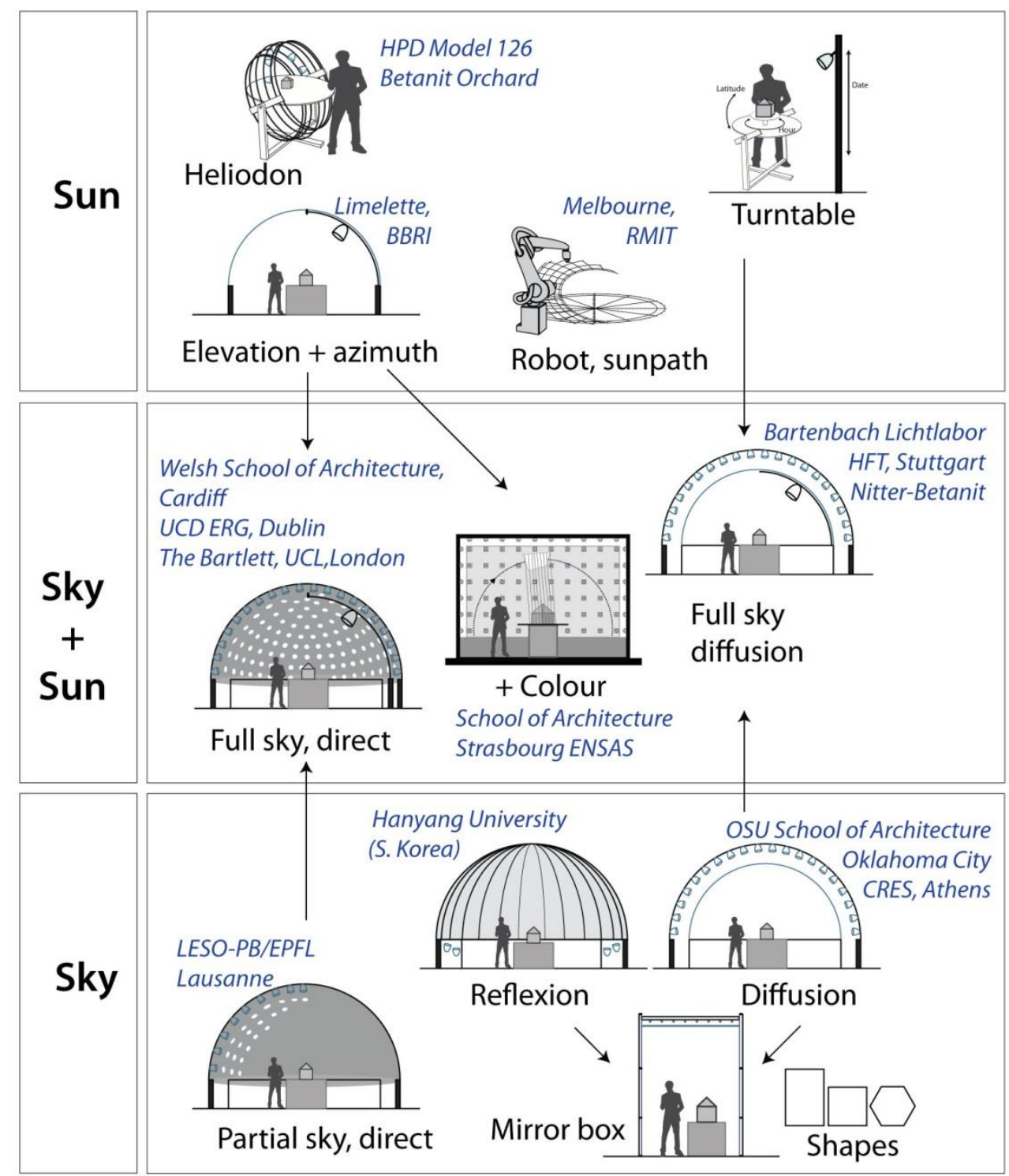

Figure 2: Daylight simulators, state of the art

The previous figure presents all the daylight simulators by organizing them in three groups depending on the lighting emission they reproduce: only the sunlight, only the light emitted by the sky, the light emitted by both the sun and the sky. Considering that the real sky emits a diffuse light, the most realistic principle is a sky working by diffusion.

Light sources are disposed behind a diffusing material. Usually artificial skies have spherical shapes [5]. The most realistic systems combine an artificial sky and a mechanical sun.

\section{PRINCIPLE AND GENERAL IMPLEMENTATION}

The experiments can be observed directly by the users from the outside of the simulator through a large opening at north. A bright awning compensates the loss of light due to this opening. The simulation is recorded by cameras positioned inside and around the architectural model. 7,000 RGB LED sources are distributed on the walls and ceiling behind polycarbonate diffusing panels. The software calculates in real-time the image of the sky and through a pixel mapping process the RBG values are applied to each LED source, so we can simulate several sky models. 


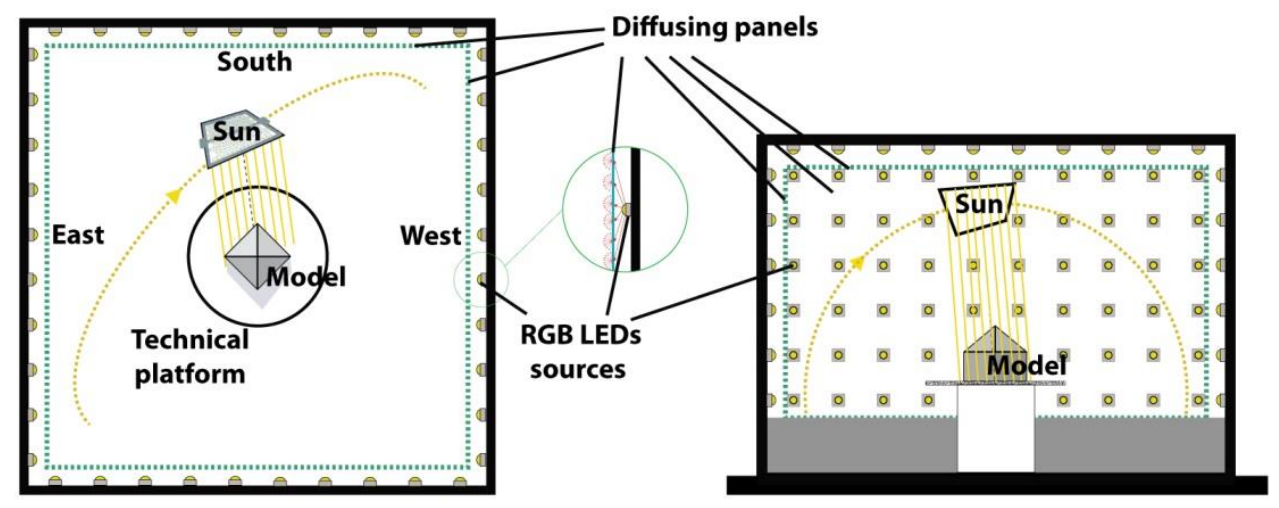

Figure 3: The functioning principle of the artificial sun \& sky

The artificial sun allows study of the light contribution inside the model and the generated shadows. The artificial sun illuminates a surface of one square meter to light $800 \mathrm{~mm}$ x 800 x 400 models with RGBW LED sources. The mechanical sun is integrated inside the artificial sky. The mechanical sun follows a semi-spherical trajectory of two meters' radius.

To obtain a lambertian diffusion for the artificial sky, we had to find the optimal diffusing material and the right distance between the light sources and this material. Because LED technology offers a possible control of each source, it is no longer necessary to build the sky with a dome shape; therefore we chose a cubic shape. After calibration in such a sky, the luminous flux of each source will have the same contribution to the centre of the experimental platform.

\subsection{Diffusing panels scattering characterization}

To characterize the diffusion of a material it is necessary to determine its transmission factor depending on the angle of observation. The source used is a He-Ne continuous laser $(\mathrm{P}=3 \mathrm{~mW}$ and $\lambda=632.8 \mathrm{~nm})$.
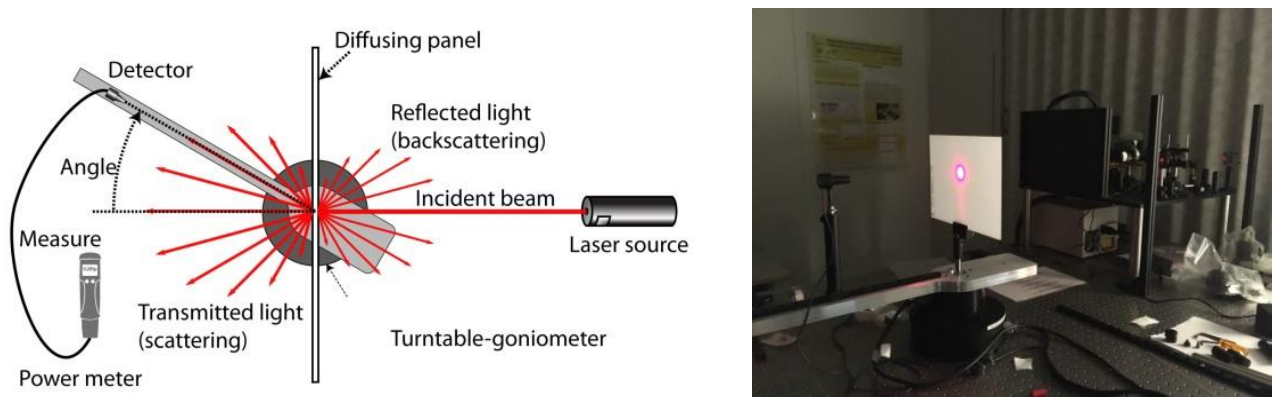

Figure 4: Experimental set-up for scattering characterization

The laser beam is perpendicular to the sample, and a power meter is disposed on a computer controlled mobile arm.

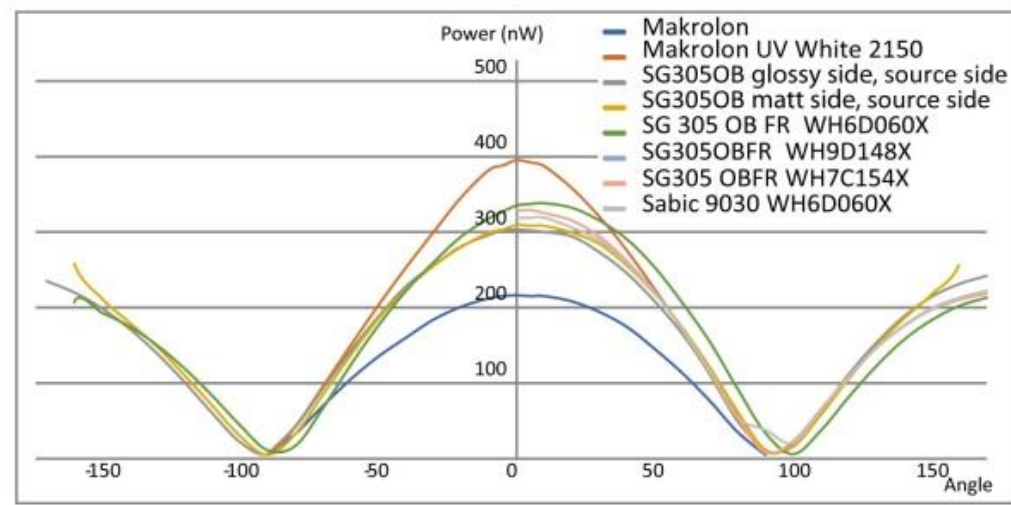

Figure 5: Samples scattering characterization. Measurement of the transmitted power depending on the angle of emission 
Scattering is a balance between light transmission at $0^{\circ}$ and transmission for higher angle values. To limit the light losses, the selected material should not be too absorbent. Theoretically a perfect diffuser commonly qualified as Lambertian is a material through which its illuminance is angularly uniform. The intensity value I depending on the angle of observation $\theta$ for a Lambertian diffuser is defined by the following equation:

$$
I=I_{0} \cos \theta
$$

The objective of these measurements is to identify which sample offers the closest characteristics to a lambertian diffuser (2). Finally the SG3050B FR Lexan polycarbonate diffusing panel with colour reference WH7C154X has been selected.

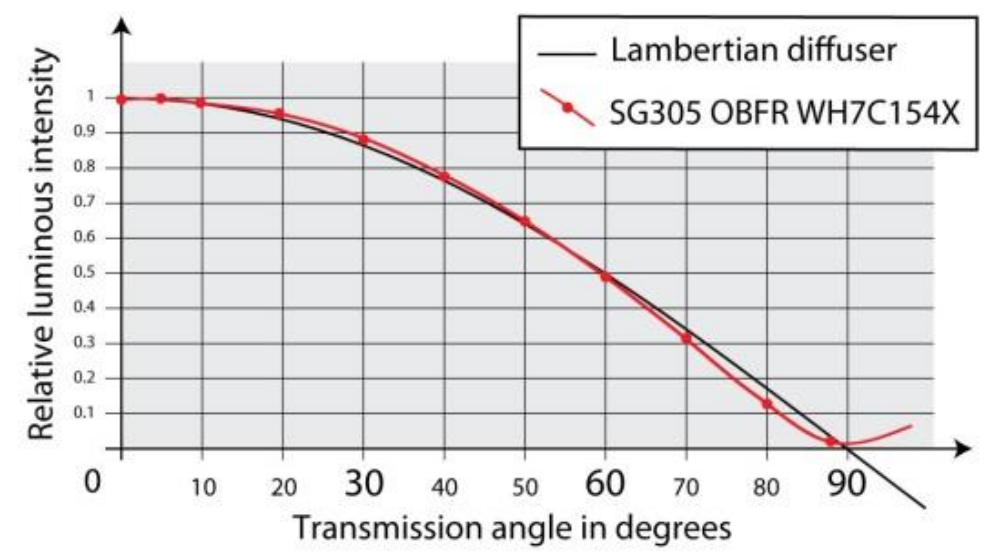

Figure 6: Lambertian diffuser versus Lexan SG 305 OBFR WH7C154X sample

\subsection{Pixel mapping}

In the simulator, each LED source composing the sky is individually controlled via a pixel mapping process using 17 Arduino devices. We developed the software able to compute the images of the sky. Knowing the position and the address of each source it is possible to consider our artificial sky as the display system for these images.

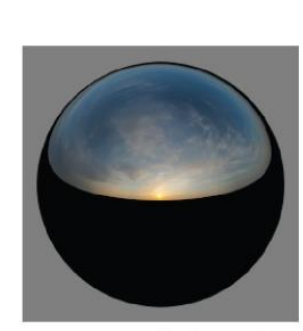

Image of the sky

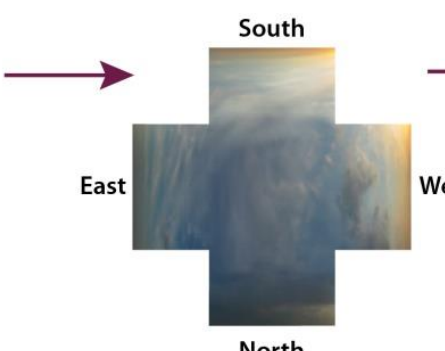

North

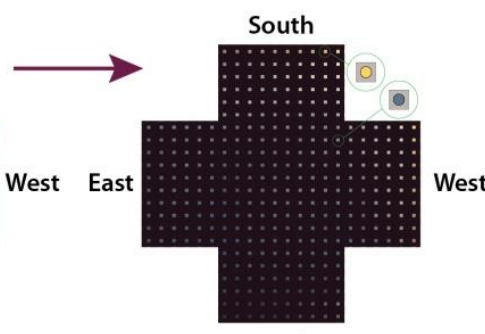

North

\subsection{Sky models}

Figure 7: pixel mapping principle

Our sky can be compared to a virtual reality CAVE.

We implemented several sky models: luminance models and colour appearance models. The computed images of the sky are displayed in real-time. The luminance models we can display are uniform luminance, overcast sky, clear sky, and all-weather models defined by Perez [6] and the CIE [7]. Appearance models are typically used in computer graphics software and games to produce realistic backgrounds. The most elaborate models (Nishita, Preetham, Haber, Bruneton, Hosek-Wilkie, etc.) integrate the physical behaviour of light in the Earth's atmosphere (turbidity, albedo, etc.). 

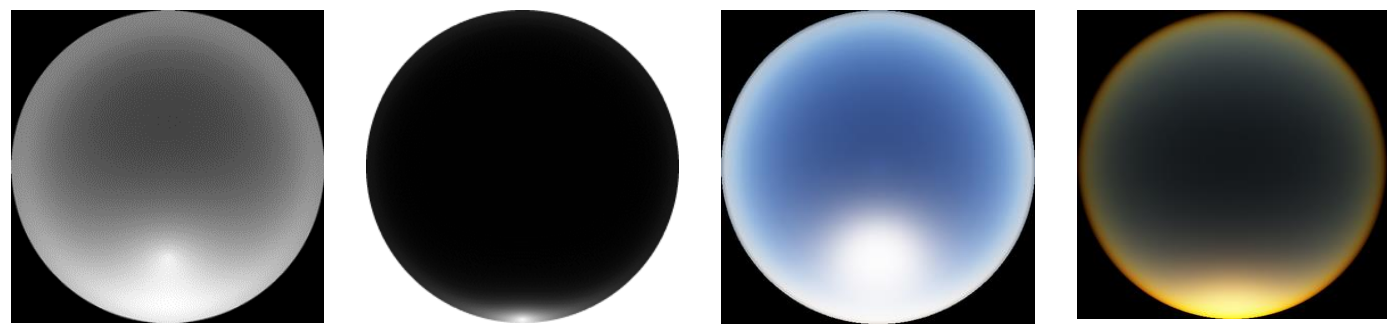

Figure 8: CIE Overcast sky at midday (left) Sky image at midday by Haber and sunset image by Nishita (right)

Appearance models are in colour and based on the results of the Perez luminance models. In our case appearance models appear to be the most interesting to use because the presence of colours increases the realism of the simulation.

\subsection{Overall functioning}

Two computers are used to control and synchronize all the components of the sun \& sky simulator, one sends all the information needed to achieve the experiment, the other one collects the experiment data and gives in real time a visual feedback.

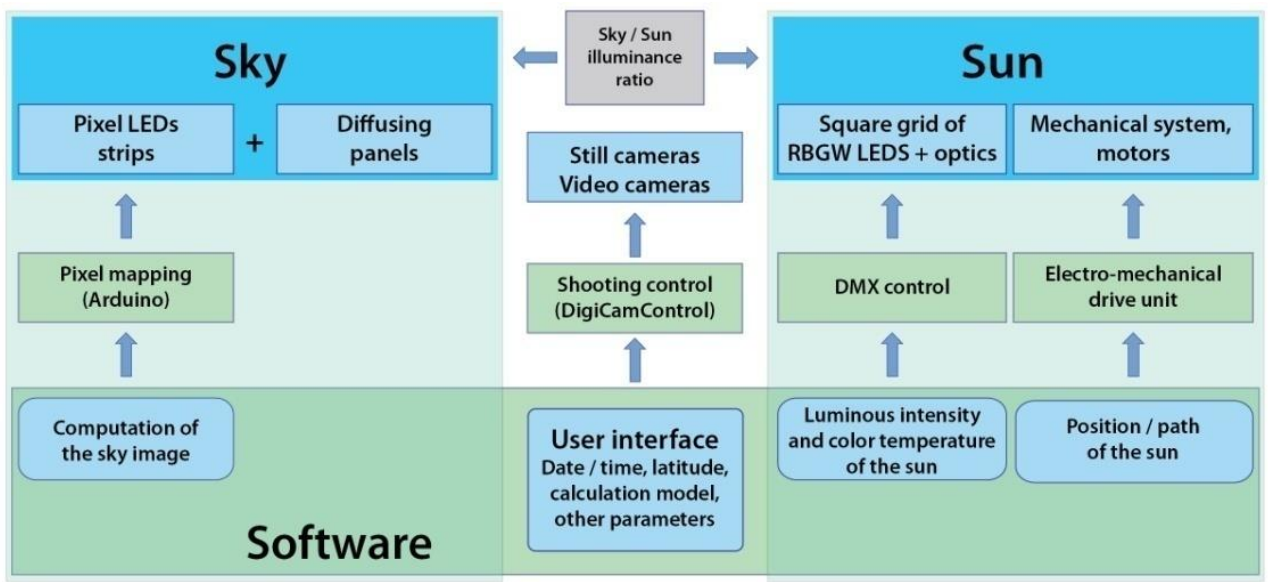

Figure 9: Software functional chart

\section{CALIBRATION}

The figure below shows in green the real position of some light sources in the cubic shape of the simulator and their projection (in red) on the theoretical spherical shape of the sky. The calibration process consists in applying a correction table to each source of the sky. So the lighting contribution of each pixel at the centre of the technical platform must be the same.

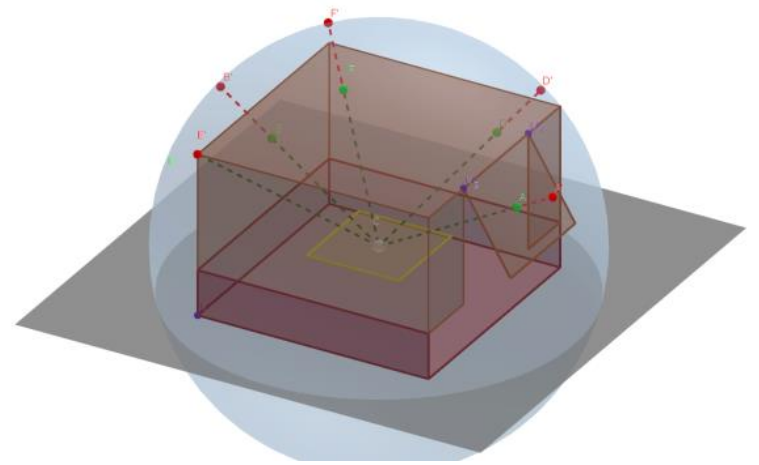

Figure 10: Theoretical position of each LED source for calibration process 


\subsection{Presentation of the correction function}

The light contribution of a pixel at the centre of the technical platform changes with the distance squared. The closest points are at the centre of each wall, while the farthest points are in the upper corners.

The luminous intensity $\mathrm{I}_{\mathrm{p}}$ of a pixel $\mathrm{p}$ in the simulator is defined by the following correction function equation:

$$
I_{p}=I_{\max }\left(\frac{D}{D_{\max }}\right)^{2}
$$

Where:

$\mathrm{D}$ is the distance between the pixel and the centre of the experimental platform

$\mathrm{D}_{\max }$ is the distance between the pixel in the upper corner and the centre of the experimental platform $\mathrm{I}_{\max }$ the intensity of the pixel in the upper corner of the simulator

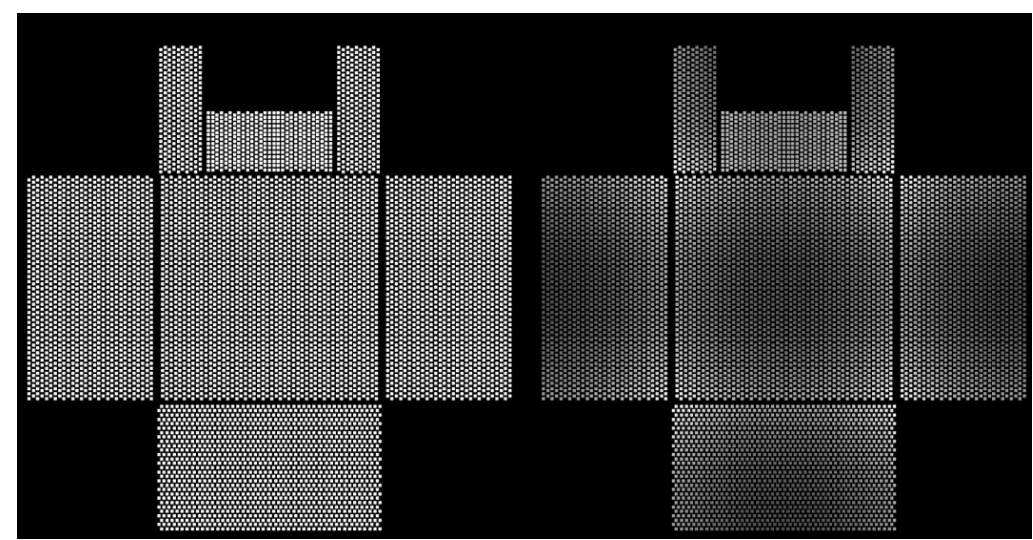

\subsection{Illuminance analysis}

Figure 11: Images of the sky displayed in the simulator without (left) and with (right) correction.

Several pictures have been taken with Canon EOS equipped with a Samyang fisheye lens. The camera is disposed at the centre of the technical platform. RGB images are then converted in false colour images to help analyse the applied corrections defined by equation (3).
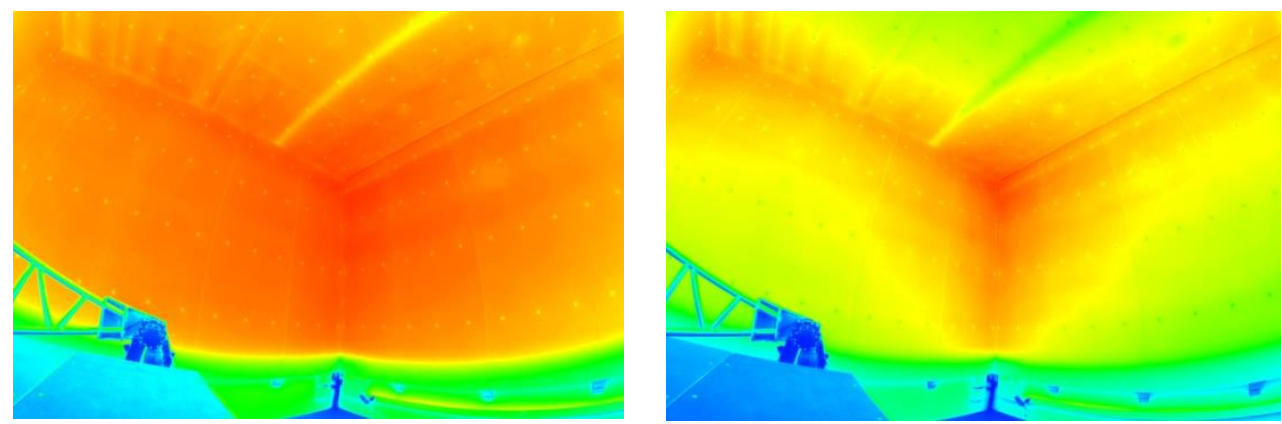

Figure 12: Picture of the South West corner, without (left) and with (right) correction

The figure above shows the light intensity without and with correction. The regular low intensity points on the pictures are associated to the polycarbonate screws use to fix the diffusing panel in front of the light sources. As expected, the intensity in centres of the walls is lower, but it seems that the contrast has been accentuated.

This unexpected result will be verified with illuminance measurements. All the next results have been measured from the centre of the platform, by varying the angle of observation. At $0^{\circ}$ and $90^{\circ}$ the sensor points toward the corners, at $45^{\circ}$ the sensor points toward the middle of the wall. 


\subsection{Illuminance measurements in the horizontal plane}

By using a goniometer disposed at the centre of the simulator on which an illuminance sensor has been attached, we can precisely measure the illumination depending on the angle of observation. A cylinder of $30 \mathrm{~cm}$ long and of a diameter of $2.4 \mathrm{~cm}$ is used to collect light in a limited angle of 4.6 degrees. The measurements are taken using a PRC Krochmann lux meter. The sensor is controlled by computer. To ensure precise measurements, the sensor performs for each position five measurements in five seconds. The five measures are then averaged. The angle values of the goniometer vary between $0^{\circ}$ and $90^{\circ}$.

The first experiment consists in measuring the illuminance of the south wall of the simulator with and without the correction function.
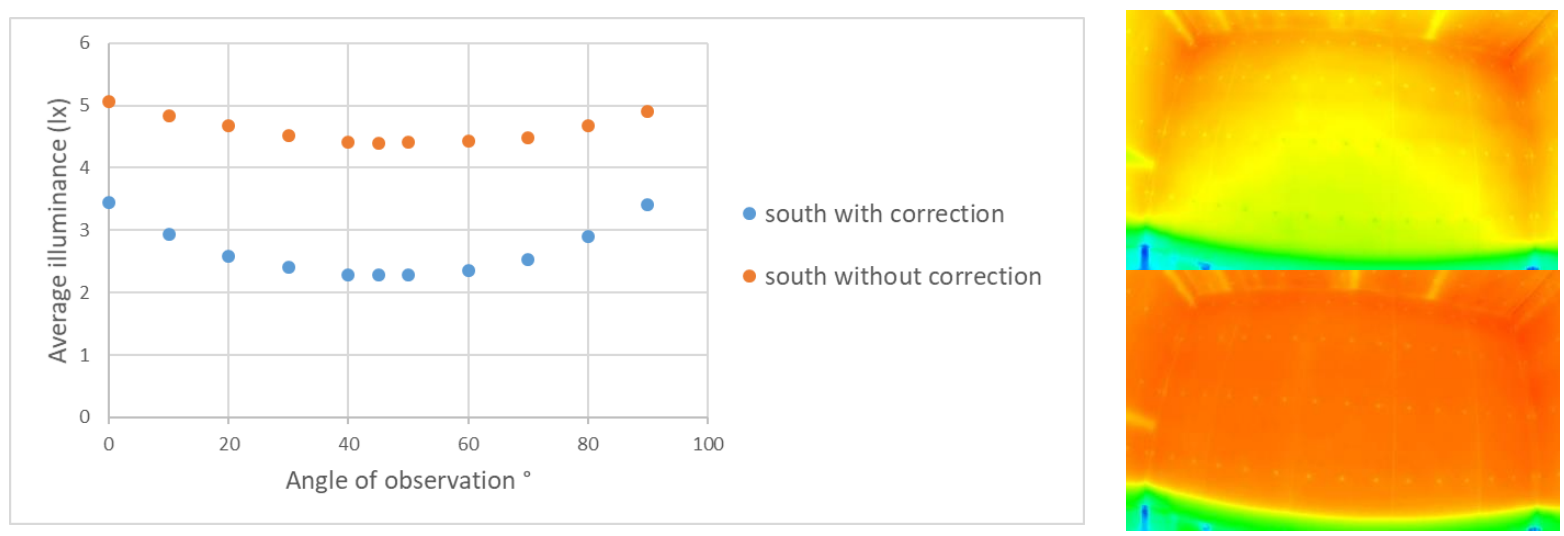

Figure 13 : The south wall illuminance with and without correction, all the walls lit

Due to the cubic shape of the simulator we expected to measure a lower illuminance in the angles compared to the illuminance measured in the middle of the wall. The figure above shows the totaly opposite of what we expected. The maximun values are measured in the corners $\left(0^{\circ}\right.$ and $\left.90^{\circ}\right)$ and the minimum appears to be for the centre of the wall $\left(45^{\circ}\right)$. The correction function decreases the illuminance but enhances the contrast. Finally the illuminance is more uniform without the correction function.

To verify these unexpected results we achieved the same measurements for the east wall. The following figure is the comparison between south and east walls illuminance.

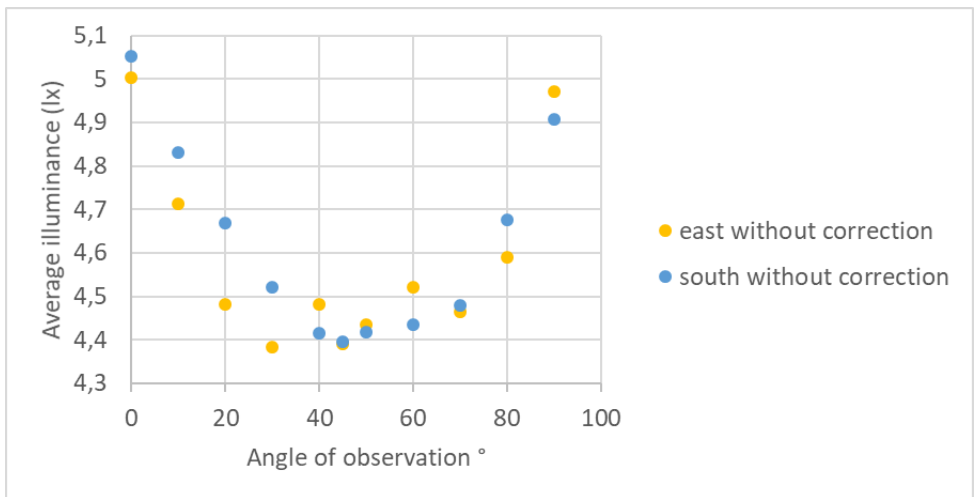

Figure 14: Comparison of the south and east walls illuminance, without correction, all the walls lit

For the two walls the profile of the variation of the illuminance is about the same with a maximun in the corners and a minimum for the centre of the wall. The difference between the two curves seems to be due to the north opening. What we measure does not only correspond to the illuminance of the studied wall but it also includes a reflected component coming from the other walls. 
To quantify the effect of the reflection on a wall, we studied the illuminance on the east wall in two different conditions: all the walls of the simulator lighted and only the east wall lighted.

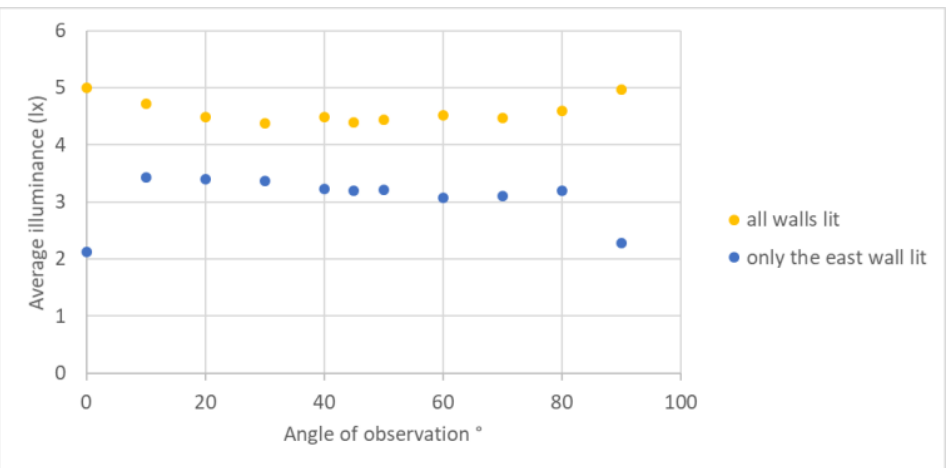

Figure 15: Comparison of the east wall illuminance measured, without correction, with only the east wall lit and all walls lit

The gap of $1.25 \mathrm{~lx}$ between the two curves is due to the reflexions of light coming from the other walls. When only one wall is lit, the illuminance in the corners is minimal because of the absence of light contribution from the adjacent walls. This is why the first and the last point of this set of values are not taken into account in the calculation of the difference between the two curves. The reflections contribute to about $20 \%$ of the measured illuminance.

\subsection{Sun illumation measurements at the surface of the experimental platform}

In reality, the direct sunlight contribution in summer is about $120000 \mathrm{~lx}$, while a blue sky is about $20000 \mathrm{~lx}$, that lead to an illuminance ratio equal to 6 . Because it is very difficult to reach such a level of illumination for the artificial sun, the most important criterion for our simulator is to reach the correct ratio value.

The sun is simulated with a high power RGBW LED panel, where 10x10 light sources are disposed on a square of onemeter size. Each LED is equipped with a total internal reflector (TIR) optics with a beam angle of $7^{\circ}$ (FWHM). With such beams the artificial sun illuminates a surface of one square meter. The sun is positioned at the zenith and sensors measure the illuminance on the technical platform. We then achieved the same measurement by lighting only the sky. We adjusted the power of the sun to respect the illuminance ratio.
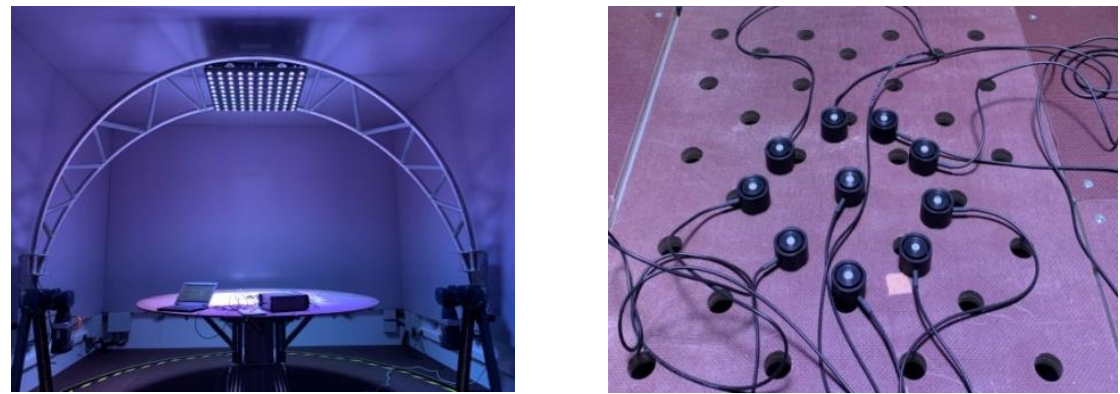

Figure 17: Sun at zenithal position and example of sensors disposition

\section{CONCLUSION}

The calibration process consists in addressing to each pixel the correct intensity in order to behave as if the sky had a spherical shape. The objective was to define the correction table to be applied to the artificial sky. After calibration, the luminous flux of each source has the same contribution to the centre of the experimental platform. Initial results have shown that the application of a theoretical correction function does not provide a uniform illuminance. 


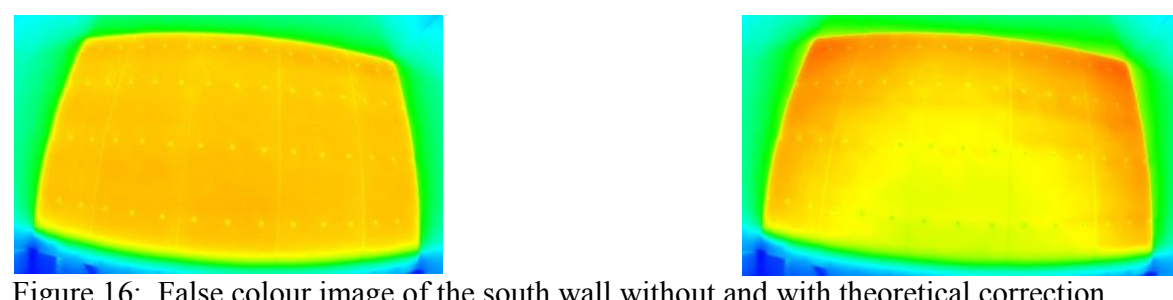

These counter-intuitive results (Figure 18) show that illuminance is uniform without applying the correction function. The inter-reflection between the walls which represent about $20 \%$ of the illuminance measured inside the simulator impose to decrease up to $10 \%$ the intensity towards the corners. Once the calibration achieved, the contribution of the sun and the sky were measured in order to adapt the intensity of the artificial sun to respect the lighting ratio of 6 .

\section{REFERENCES}

[1] Richard Kittler, Stanislav Darula, Determination of time and sun position system, Solar Energy, Volume 93, 2013, Pages 72-79, ISSN 0038-092X

[2] D. Croghan, Daylighting by Design: 2. Some early examples of the use of an artificial sky, (2015) (unpublished paper, retrieved on ResearchGate)

[3] Nawab, M. (1981). DEVELOPMENT AND USE OF A HEMISPHERICAL SKY SIMULATOR. Lawrence Berkeley National Laboratory. Report \#: LBL-13400.

[4] Okado, Masaaki \& Nakamura, Hiroshi \& Koga, Yasuko \& Goto, Koichi \& Fujii, Shun'ichi. (1997). Development of an Artificial Sky: part 1: Various Artificial Skies in the World. Journal of the Illuminating Engineering Institute of Japan.

[5] Maria K. VRONTISSI, Designing and building a geodesic dome as a bearing structure for an 'artificial sky' lighting installation, Proceedings of the International Association for Shell and Spatial Structures (IASS) Symposium 2009, Valencia

[6] Perez, R., Seals, R., \& Michalsky, J. (1993). All-weather model for sky luminance distribution-preliminary configuration and validation. Solar energy, 50(3), 235-245.

[7] Darula, S., \& Kittler, R. (2002). CIE general sky standard defining luminance distributions. Proceedings eSim, 11-13. 